\title{
Vortex arrays for sinh-Poisson equation of two-dimensional fluids: Equilibria and stability
}

\author{
D. Gurarie ${ }^{a)}$ \\ Department of Mathematics, Case Western Reserve University, Cleveland, Ohio 44106 \\ K. W. Chow ${ }^{\text {b) }}$ \\ Department of Mechanical Engineering, University of Hong Kong, Pokfulam Road, Hong Kong
}

(Received 12 November 2003; accepted 21 May 2004; published online 23 July 2004)

\begin{abstract}
The sinh-Poisson equation describes a stream function configuration of a stationary two-dimensional (2D) Euler flow. We study two classes of its exact solutions for doubly periodic domains (or doubly periodic vortex arrays in the plane). Both types contain vortex dipoles of different configurations, an elongated "cat-eye" pattern, and a "diagonal" (symmetric) configuration. We derive two new solutions, one for each class. The first one is a generalization of the MallierMaslowe vortices, while the second one consists of two corotating vortices in a square cell. Next, we examine the dynamic stability of such vortex dipoles to initial perturbations, by numerical simulations of the 2D Euler flows on periodic domains. One typical member from each class is chosen for analysis. The diagonally symmetric equilibrium maintains stability for all (even strong) perturbations, whereas the cat-eye pattern relaxes to a more stable dipole of the diagonal type.
\end{abstract}

(C) 2004 American Institute of Physics. [DOI: 10.1063/1.1772331]

\section{INTRODUCTION}

Any stationary two-dimensional (2D) Euler flow, free of body forces, has stream field $\psi$ and vorticity $\zeta$ satisfying the functional relation,

$$
\zeta=\nabla^{2} \psi=\psi_{x x}+\psi_{y y}=f(\psi) .
$$

Our convention for $2 \mathrm{D}$ velocity here is $(u, v)=\left(-\psi_{y}, \psi_{x}\right)$. The function $f$ could be arbitrary, but in many statistical theories of $2 \mathrm{D}$ turbulence in fluids and plasmas, and numeric simulations $^{1-10}$ it appears in the form of a hyperbolic sine,

$$
\nabla^{2} \psi=f(\psi)=-\sigma \sinh \psi, \quad(\sigma>0),
$$

called the sinh-Poisson equation.

The sinh-Poisson equation is closely related to some known integrable soliton models, and has a number of exact solutions important in theoretical studies and applications. A well-known solution of (2) is the array of counterrotating Mallier-Maslowe vortices, ${ }^{2-5}$ as well as doubly periodic solutions (in the $x$ and $y$ directions). ${ }^{6-12}$

Our goal here is twofold. We shall review the known vortex arrays (Classes I and II, Sec. II) and derive two new solutions (Sec. III). The first one is a doubly periodic generalization of the Mallier-Maslowe vortices. The second one consists of two corotating vortices in a square cell. Second, we shall examine the dynamic stability of vortex equilibria using a numeric (semi-Lagrangian) code to simulate 2D Euler flows, and we take one example from each class as an illustration (Sec. IV).

\footnotetext{
a) Telephone: (216) 368-2857; fax: (216) 368-5163. Electronic mail: dxg5@case.edu

${ }^{b)}$ Author to whom correspondence should be addressed. Telephone: (852) 2859 2641; fax: (852) 2858 5415. Electronic mail: kwchow@hkusua.hku.hk
}

The first example is a known solution from Class II and the second example is the new solution for Class I. For convenience we shall term them the sn-sn and sn-dn dipoles based on their analytical structure, or alternatively, vortex solitons, not in a strict mathematical sense, but by loose association with soliton-style type models and expansion used in their derivation. Both examples consist of vortex dipoles on a fundamental doubly periodic domain, but they differ in their configuration and the vortex shape: elongated (cat-eye) soliton versus a diagonally symmetric type.

We study the dynamic stability of both vortex arrays (or vortex solitons) to initial perturbations. The diagonally symmetric configuration proves to be structurally stable to any (high level) noise, and relaxes to a similar dipole state. We confirm it by the analysis of the $\psi-\zeta$ scatter plot, and by further comparison of the dynamic equilibrium with the exact analytic solution. The cat-eye soliton proves to be unstable (even to small perturbations), and relaxes after a transitory phase to a diagonally symmetric (near-linear) soliton state.

The relaxation of 2D Navier-Stokes flows to large-scale equilibrium states was observed in several works. ${ }^{1,3,13-18}$ There were also analytic studies based on the Arnold stability criteria, e.g., Mallier-Maslowe or Stuart vortices. ${ }^{4,5}$ Our work focuses on dynamic stability for doubly periodic vortex arrays for the sinh-Poisson equation.

\section{BACKGROUND ON VORTEX EQUILIBRIA}

Our classification scheme is based on the following mathematical features and analytic formulation, as well as the resulting flow pattern:

(I) The first category of doubly periodic arrays of vorti- 


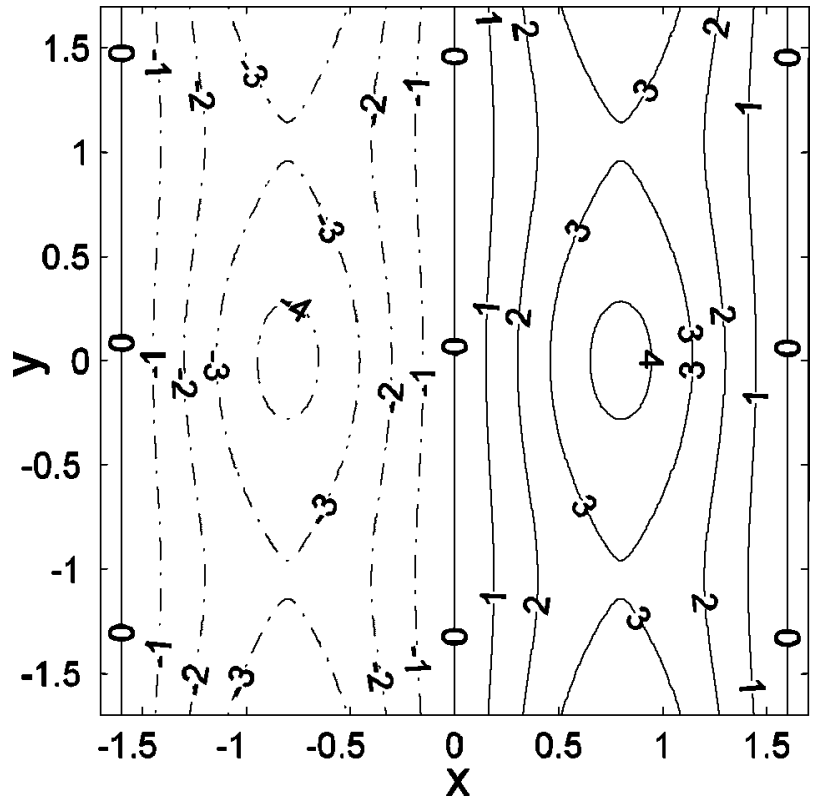

FIG. 1. Streamlines of the vortical pattern defined by Eqs. (9) and (10): $r$ $=2.1082, s=1.6667, k=0.5, k_{1}=0.6$.

ces can be expressed in terms of products of elliptic functions, ${ }^{8}$

$$
\begin{aligned}
& \psi=4 \tanh ^{-1}\left[\left(\frac{s k_{1}}{r \sqrt{1-k^{2}}}\right) \operatorname{cn}(r x, k) \operatorname{cn}\left(s y, k_{1}\right)\right], \\
& r^{4} k^{2}\left(1-k^{2}\right)=s^{4} k_{1}^{2}\left(1-k_{1}^{2}\right), \\
& r^{2}\left(1-2 k^{2}\right)+s^{2}\left(1-2 k_{1}^{2}\right)=\sigma .
\end{aligned}
$$

$k, k_{1}$ are distinct, independent parameters (moduli) of the Jacobi elliptic functions.

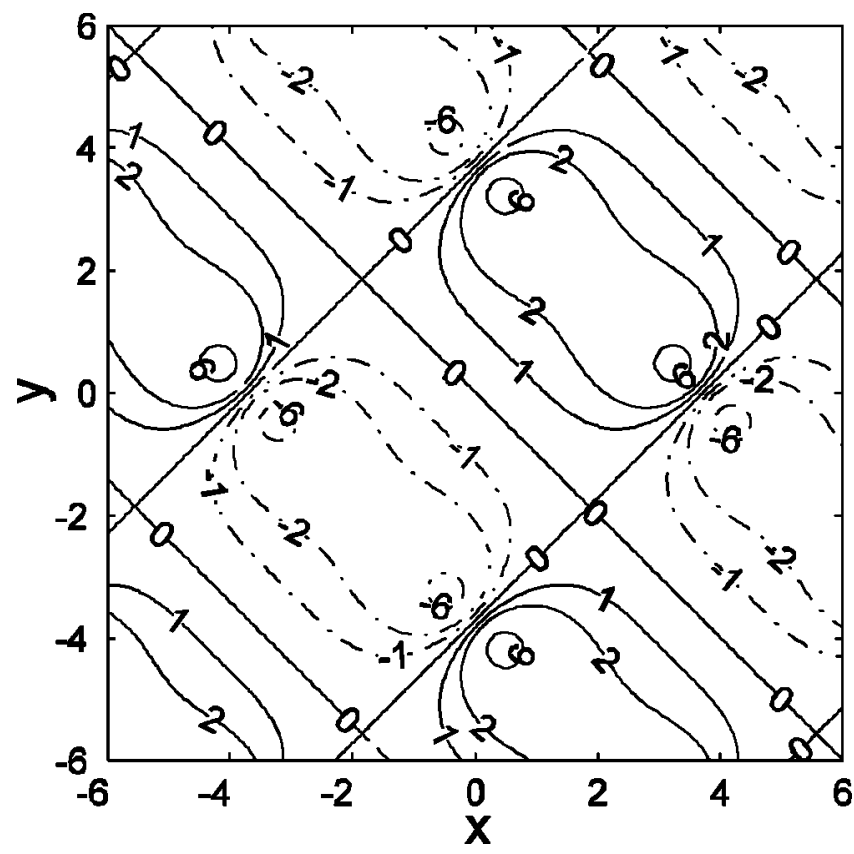

FIG. 2. Streamlines of the vortical pattern defined by Eq. (14): $r=1, A$ $=0.4$.

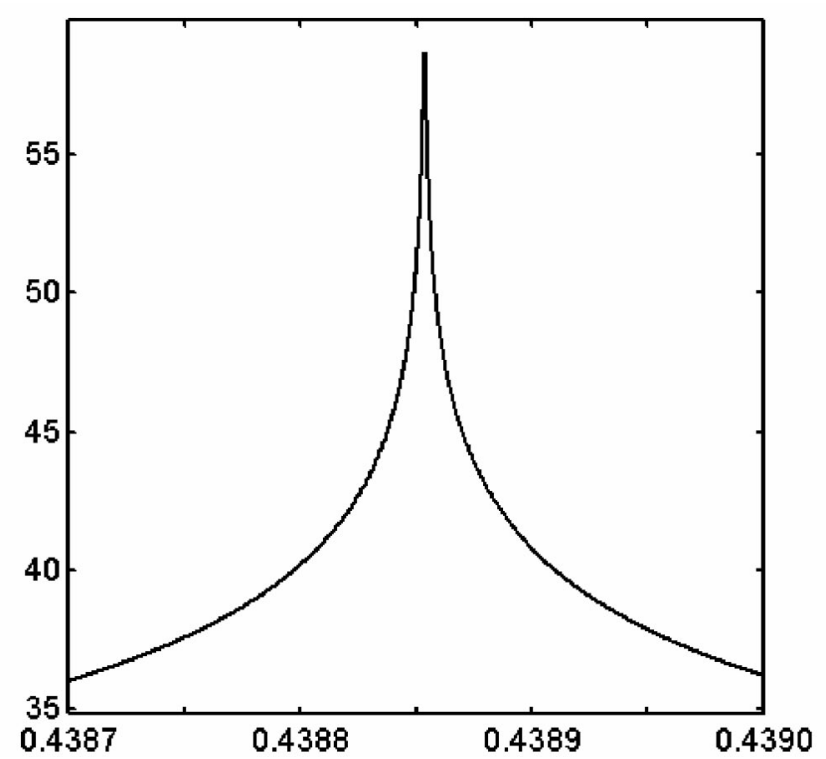

FIG. 3. Values of the stream function of Eq. (14) vs $x$ near a "point singularity," $r=1, A=0.4$.

(II) The second category of doubly periodic patterns can be expressed in terms of rational expressions of (usually linear or quadratic products of) elliptic functions. ${ }^{6,9,10}$

In the first subdivision the flow will consist of a sequence of cat-eye patterns (except for some special parameter values).

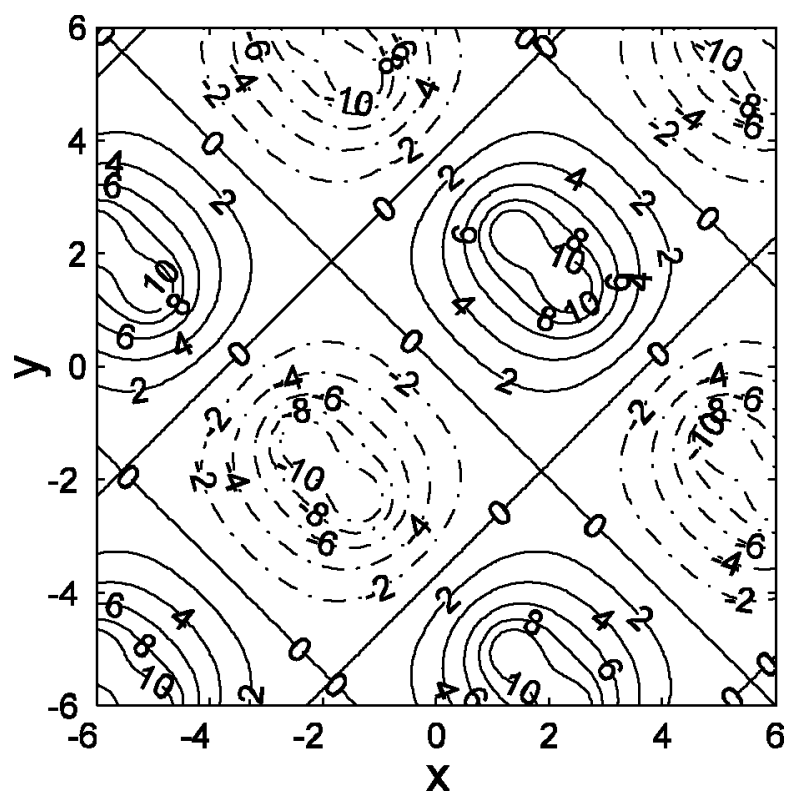

FIG. 4. Streamlines of the vortical pattern of Eq. (14) for a larger value of $A=0.7, r=1$. 
0.05
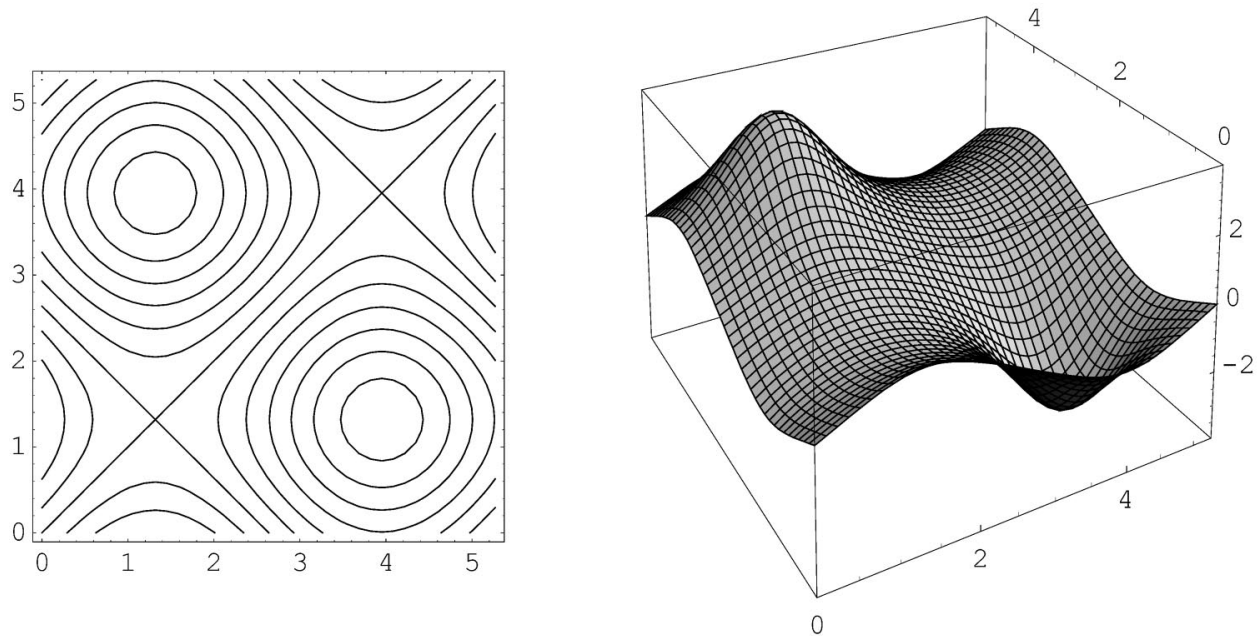

0.15
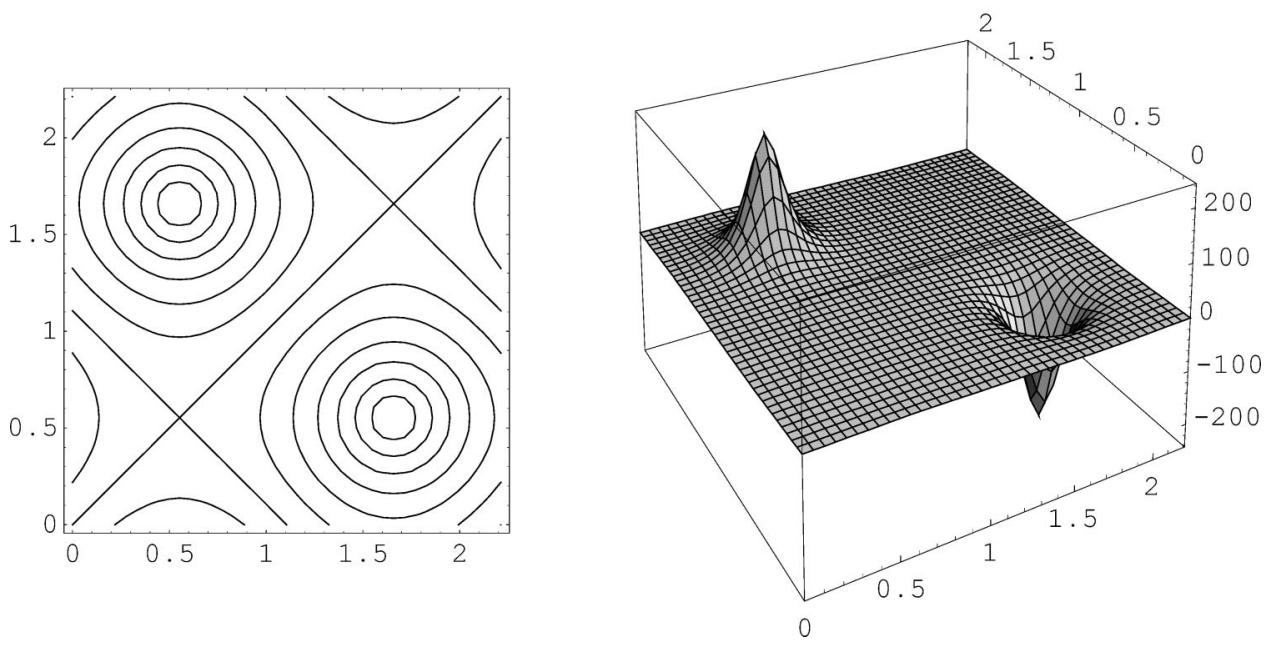

FIG. 5. sinh-Poisson vortex dipoles of Eq. (5): stream function (left) and vorticity (right) at two values of the modulus: $k=0.05$ (above) and $k=0.15$ (below).

(a)

(c)

$$
\begin{aligned}
& \psi=4 \tanh ^{-1}\left[\frac{\operatorname{dn}(r x, k) \operatorname{dn}\left(s y, k_{1}\right)-\left(1-k^{2}\right)^{1 / 4}\left(1-k_{1}^{2}\right)^{1 / 4}}{\left(1-k_{1}^{2}\right)^{1 / 4} \operatorname{dn}(r x, k)+\left(1-k^{2}\right)^{1 / 4} \operatorname{dn}\left(s y, k_{1}\right)}\right], \\
& r\left(1+\sqrt{1-k^{2}}\right)=s\left(1+\sqrt{1-k_{1}^{2}}\right), \\
& \sigma=-r^{2}\left(1-\sqrt{1-k^{2}}\right)^{2}+4 s^{2} \sqrt{1-k_{1}^{2}} .
\end{aligned}
$$

$$
\begin{aligned}
& \psi=4 \tanh ^{-1}\left\{\frac{s\left(1-k_{1}^{2}\right)^{1 / 4}}{2 r\left(1-\sqrt{1-k^{2}}\right)}\left[\frac{\operatorname{dn}(r x, k)}{\left(1-k^{2}\right)^{1 / 4}}-\frac{\left(1-k^{2}\right)^{1 / 4}}{\operatorname{dn}(r x, k)}\right]\right. \\
& \left.\times\left[\frac{\operatorname{dn}\left(s y, k_{1}\right)}{\left(1-k_{1}^{2}\right)^{1 / 4}}-\frac{\left(1-k_{1}^{2}\right)^{1 / 4}}{\operatorname{dn}\left(s y, k_{1}\right)}\right]\right\} \text {, } \\
& s^{2}\left(1-k_{1}^{2}\right)^{1 / 4}\left[1-\sqrt{1-k_{1}^{2}}\right]=r^{2}\left(1-k^{2}\right)^{1 / 4}\left[1-\sqrt{1-k^{2}}\right],
\end{aligned}
$$

(b)

$$
\begin{aligned}
& \psi=4 \tanh ^{-1}\left[\frac{\sqrt{k} \operatorname{sn}(r x, k)-\sqrt{k_{1}} \operatorname{sn}\left(s y, k_{1}\right)}{1+\sqrt{k k_{1}} \operatorname{sn}(r x, k) \operatorname{sn}\left(s y, k_{1}\right)}\right], \\
& s^{2}\left(1-k_{1}\right)^{2}=\sigma+4 r^{2} k, \quad s\left(1+k_{1}\right)=r(1+k) .
\end{aligned}
$$

In the second subdivision the flow will consist of arrays of rectangular cells with alternating signs of vorticity.

These solutions are obtained by the Hirota bilinear method, a well-established technique in the modern theory of nonlinear waves, ${ }^{11}$ through the following intermediate steps: 

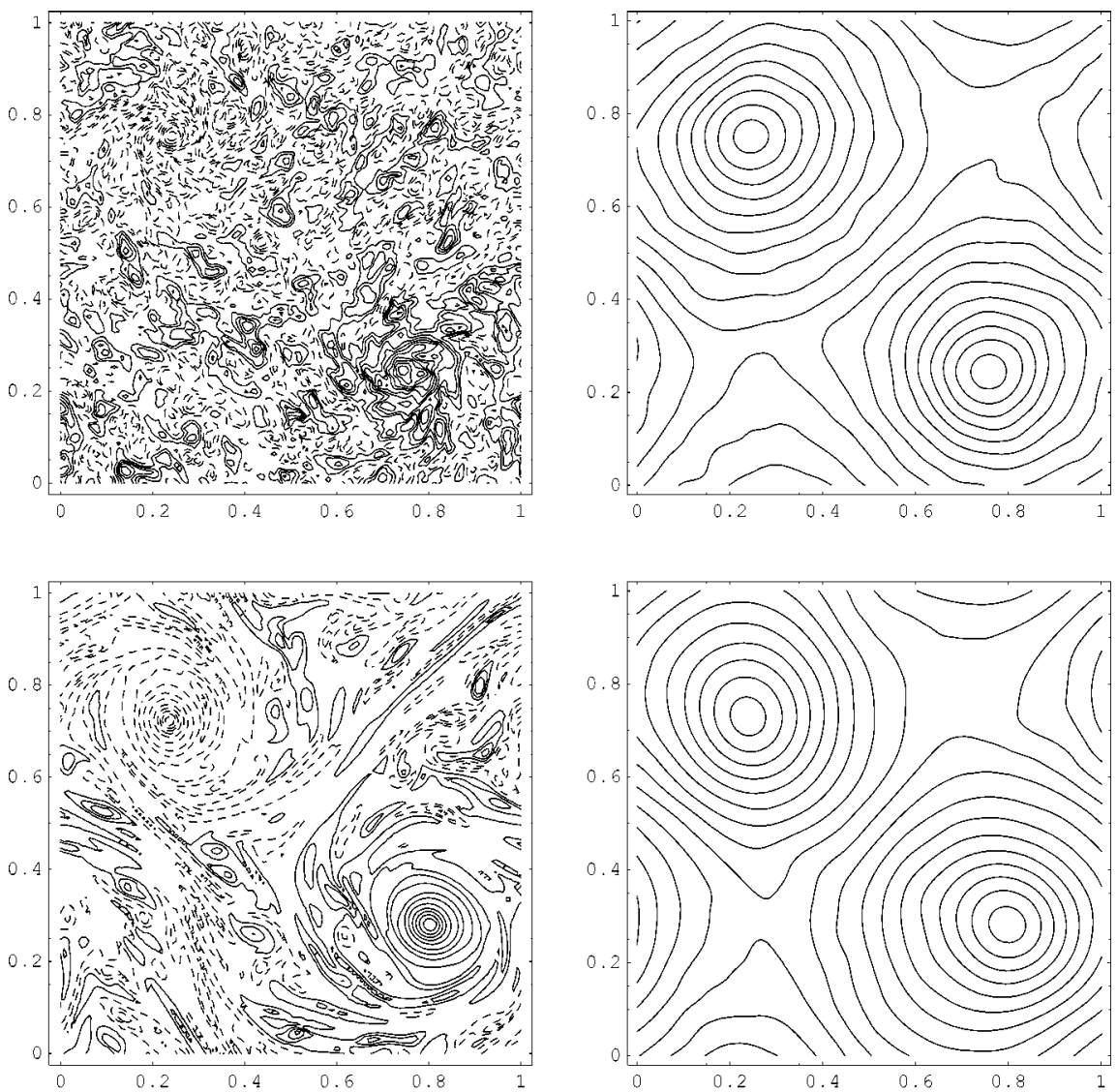

FIG. 6. Snapshots of vorticity (left panel) and stream function (right panel) at time $t=5,40$, and 120 of the eddy-turnover units $\tau=1 / \max \left|\zeta_{0}\right|$ (from top).
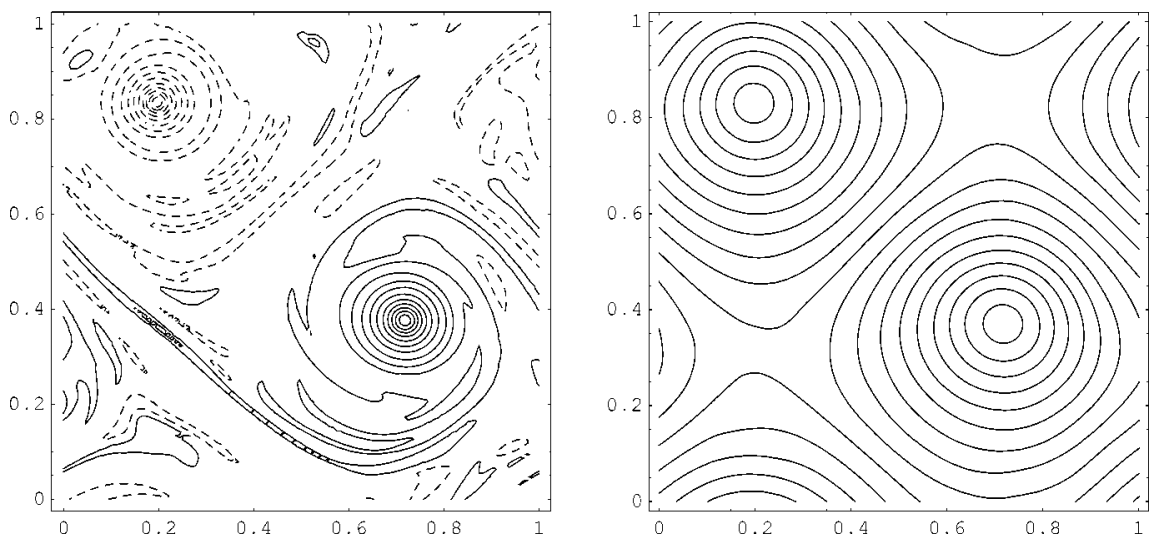

$$
\begin{aligned}
& \psi=4 \tanh ^{-1}\left(\frac{g}{f}\right), \quad\left(D_{x}^{2}+D_{y}^{2}+C\right)(g \cdot f)=0, \\
& \left(D_{x}^{2}+D_{y}^{2}+C+\sigma\right) g f=0 .
\end{aligned}
$$

$C$ is a constant and $D$ is the Hirota bilinear operator defined by

$$
\begin{aligned}
D_{x}^{m} D_{t}^{n} g \cdot f= & \left(\frac{\partial}{\partial x}-\frac{\partial}{\partial x^{\prime}}\right)^{m}\left(\frac{\partial}{\partial t}-\frac{\partial}{\partial t^{\prime}}\right)^{n} \\
& \times g(x, t) f\left(x^{\prime}, t^{\prime}\right)_{x=x^{\prime}, t=t^{\prime}} .
\end{aligned}
$$

The trick here is to simplify the Hirota derivatives through theta-function identities, ${ }^{12}$ as is documented in our earlier works. ${ }^{6,9}$ The main idea is to find combinations of theta functions where the Hirota derivatives are proportional to themselves. As an independent check, we verify the validity of the new solutions below by direct differentiation and substitution in (2) with the software MATHEMATICA.

\section{FURTHER EXACT SOLUTIONS}

Here we present a new solution of each category.

\section{A. First new solution}

Proceeding along this line of reasoning a new solution of (2) belonging to Category (I) is 


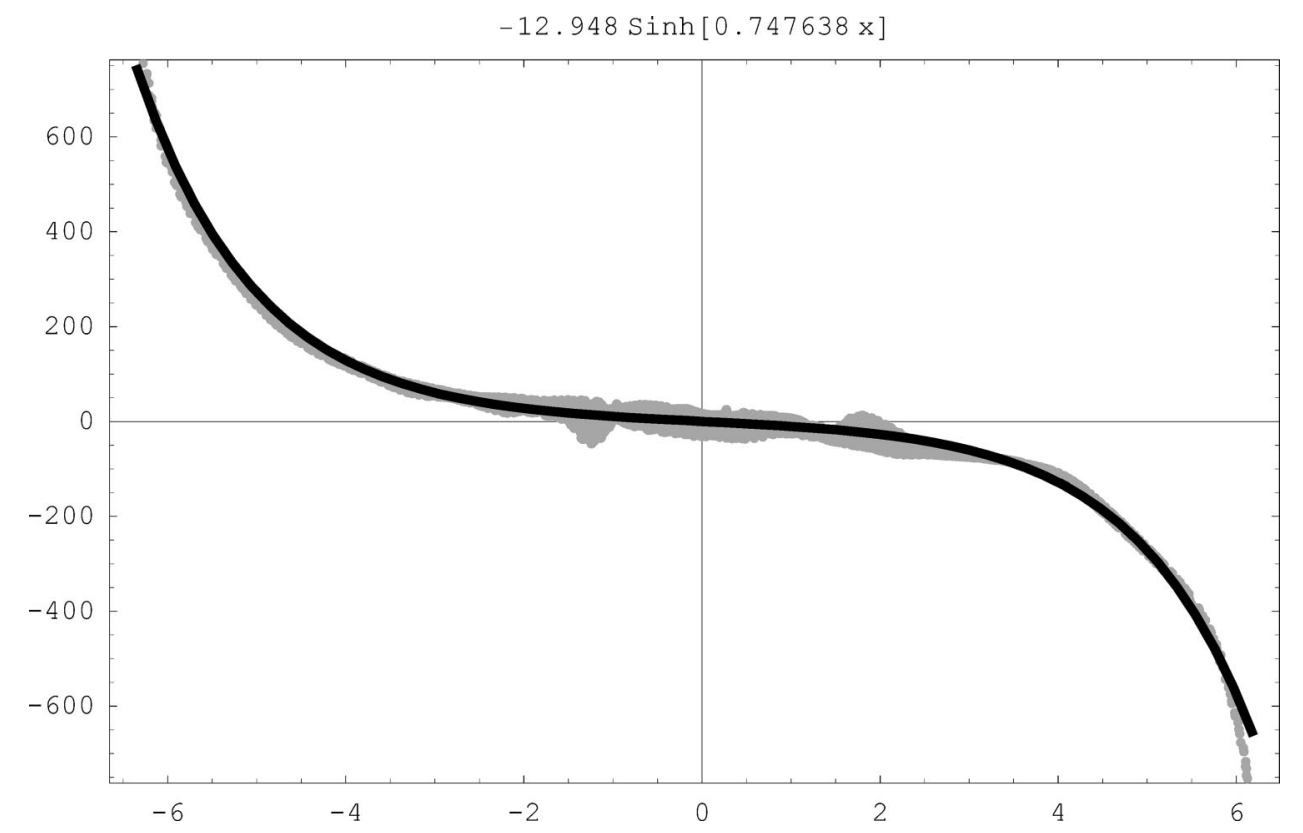

FIG. 7. Vorticity-stream scatter plot at time $=500$ and its sinh-fit profile.

$$
\begin{aligned}
& \psi=4 \tanh ^{-1}\left[\frac{\sqrt{k} \operatorname{sn}(r x, k) \operatorname{dn}\left(s y, k_{1}\right)}{\left(1-k_{1}^{2}\right)^{1 / 4}}\right], \\
& r^{2} k=s^{2} \sqrt{1-k_{1}^{2}}, \quad r^{2}\left(1+k^{2}\right)=\sigma+s^{2}\left(2-k_{1}^{2}\right) .
\end{aligned}
$$

The two expressions of (10) define the constraints relating the wave numbers $(r, s)$ and the distinct, independent moduli $\left(k, k_{1}\right)$ of the elliptic functions. The solution will definitely be nonsingular if $k<\sqrt{1-k_{1}^{2}}$. The interesting feature of (9) is the choice of the elliptic function dn, which has no real zero. In terms of flow patterns, there will be straight streamlines in one direction only, and thus the flow configuration cannot be confined to a rectangular cell (Fig. 1). Examination of Fig. 1 reveals that the Mallier-Maslowe vortices are recovered by allowing the period in the vertical direction to be indefinitely large. Mathematically, this is achieved by taking these special limits,

$$
\begin{aligned}
& k \rightarrow 0, \quad k_{1} \rightarrow 1, \quad \lim _{k \rightarrow 0} \operatorname{sn}(z, k)=\sin z, \\
& \lim _{k_{1} \rightarrow 1} \operatorname{dn}\left(z, k_{1}\right)=\operatorname{sech} z .
\end{aligned}
$$

With the balance

$$
k=\left(\frac{\alpha^{2}}{1+\alpha^{2}}\right) \sqrt{1-k_{1}^{2}}, \quad r^{2}=1+\alpha^{2},
$$

one reproduces (after a simple phase shift of $\pi / 2$ ) the Mallier-Maslowe vortices,

$$
\psi=4 \tanh ^{-1}\left[\frac{\alpha \cos \sqrt{1+\alpha^{2}} x}{\sqrt{1+\alpha^{2}} \cosh \alpha y}\right] .
$$

\section{B. Second new solution}

A new solution for category (II) is

$$
\begin{aligned}
& \psi=4 \tanh ^{-1} \\
& \times\left\{\frac{A\left[\operatorname{sn}\left(r x, k_{0}\right) \operatorname{dn}\left(r y, k_{0}\right)+\operatorname{dn}\left(r x, k_{0}\right) \operatorname{sn}\left(r y, k_{0}\right)\right]}{1+\sqrt{1-2 A^{2}} \operatorname{cn}\left(r x, k_{0}\right) \operatorname{cn}\left(r y, k_{0}\right)}\right\}, \\
& k_{0}=\frac{1}{\sqrt{2}}, \quad 0<A<\frac{1}{\sqrt{2}} \text {. }
\end{aligned}
$$

This is a one parameter $(A)$ family of solutions but only for a very special modulus of the Jacobi elliptic functions. The wave numbers in the $x, y$ directions must be identical. A straightforward calculation shows that the vorticity parameter of (2), $\sigma$, is zero (almost everywhere), and thus it is tempting to conclude that the flow is irrotational. An inspection of the flow configuration associated with (14) (Fig. 2) clearly shows otherwise. The patterns consist of square cells of alternating signs of rotation. Within each cell there are two corotating vortices. Each side of the square cell is of the length $2 \sqrt{2} K / r$, where $K$ is the elliptic integral of the first kind with modulus $k_{0}=1 / \sqrt{2}$.

$$
K\left(k_{0}\right)=K(1 / \sqrt{2})=\int_{0}^{\pi / 2} \frac{d \theta}{\sqrt{1-\left(\sin ^{2} \theta\right) / 2}} .
$$

Owing to the complexity in handling elliptic functions, the structure of the singularities is best appreciated by exam- 

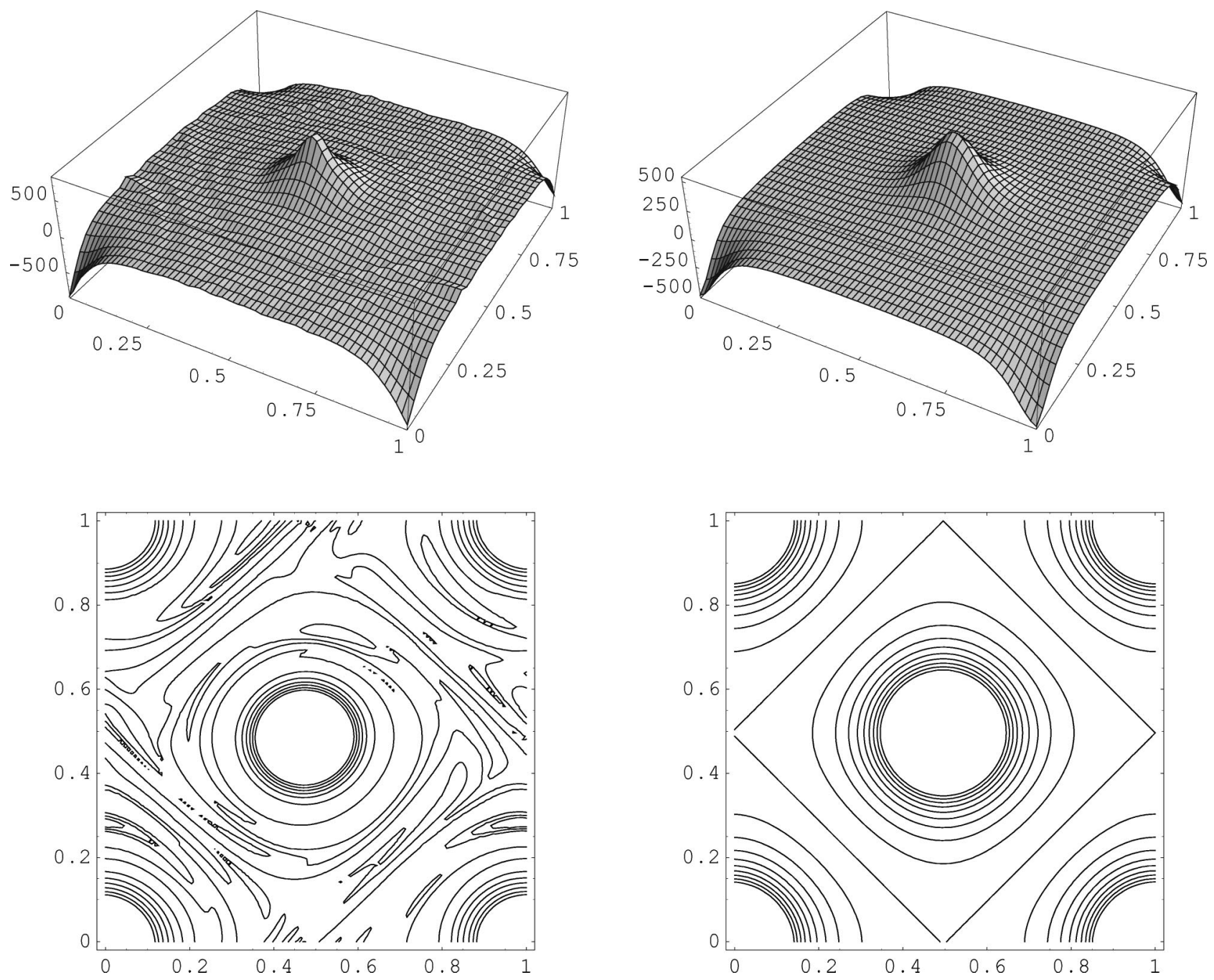

FIG. 8. Comparison between the equilibriated vorticity (left) and the sn-sn dipole with modulus $k=0.13$, estimated from the best-fit sinh plot. For the sake of comparison the vortex cores are shifted here to the center of the rectangle.

ining the flow properties. As an example, consider $A=0.4$, where one center of the vortices is determined to be at approximately $x=0.438854, y=3.269295$. The subtle feature regarding this singularity is that the stream function remains finite, but probably exhibits a cusp (Fig. 3). The velocities (derivatives of the stream function) attain large values and do display a sharp jump across the potential location of the point singularity. The situation is then very similar to a line vortex in the classical theory of potential flow, but the structure here is considerably more complex, as elliptic functions and the sinh-Poisson equation are involved. As $A$ increases from 0 to $1 / \sqrt{2}$, the locations of the two point vortices shift from the edges of the square cell to the center of the cell (Fig. 4).

A common feature for these two categories of vortical patterns is that the onset of singularities is usually associated with $\sigma=0$. The stream function usually becomes infinite at isolated points. The flow, for this special regime of parameters, becomes irrotational almost everywhere except at the point singularities. The situation for (6) of category (II) is explained in an earlier paper. ${ }^{10}$ This scenario seems to repeat for most vortical patterns here. Omitting the details, one can also show that the same fact applies to vortical patterns (4) and (5) of category (II). For (3) of category (I) and the simple case $r=s, k_{1}=\sqrt{1-k^{2}}$, the flow reduces to an array of point vortices located at where the Jacobi elliptic cosine function $\mathrm{cn}$ is one. The same reasoning applies to the new solutions (9) and (10) here, except that the point vortices now are located at where the Jacobi elliptic functions sn and dn are one.

However, these statements will make (14) even more striking, as the stream function does not seem to become infinite, and yet the velocities tend to become large. The structures need further elucidation, and await future efforts.

\section{DYNAMIC STABILITY}

To address the dynamic stability of 2D flows, a few general comments are in order. The ideal 2D Euler fluid is not a physical model that one can experiment with or test numerically. Indeed, any form of numeric discretization will produce small-scale (subgrid) mixing and/or dissipation in the 

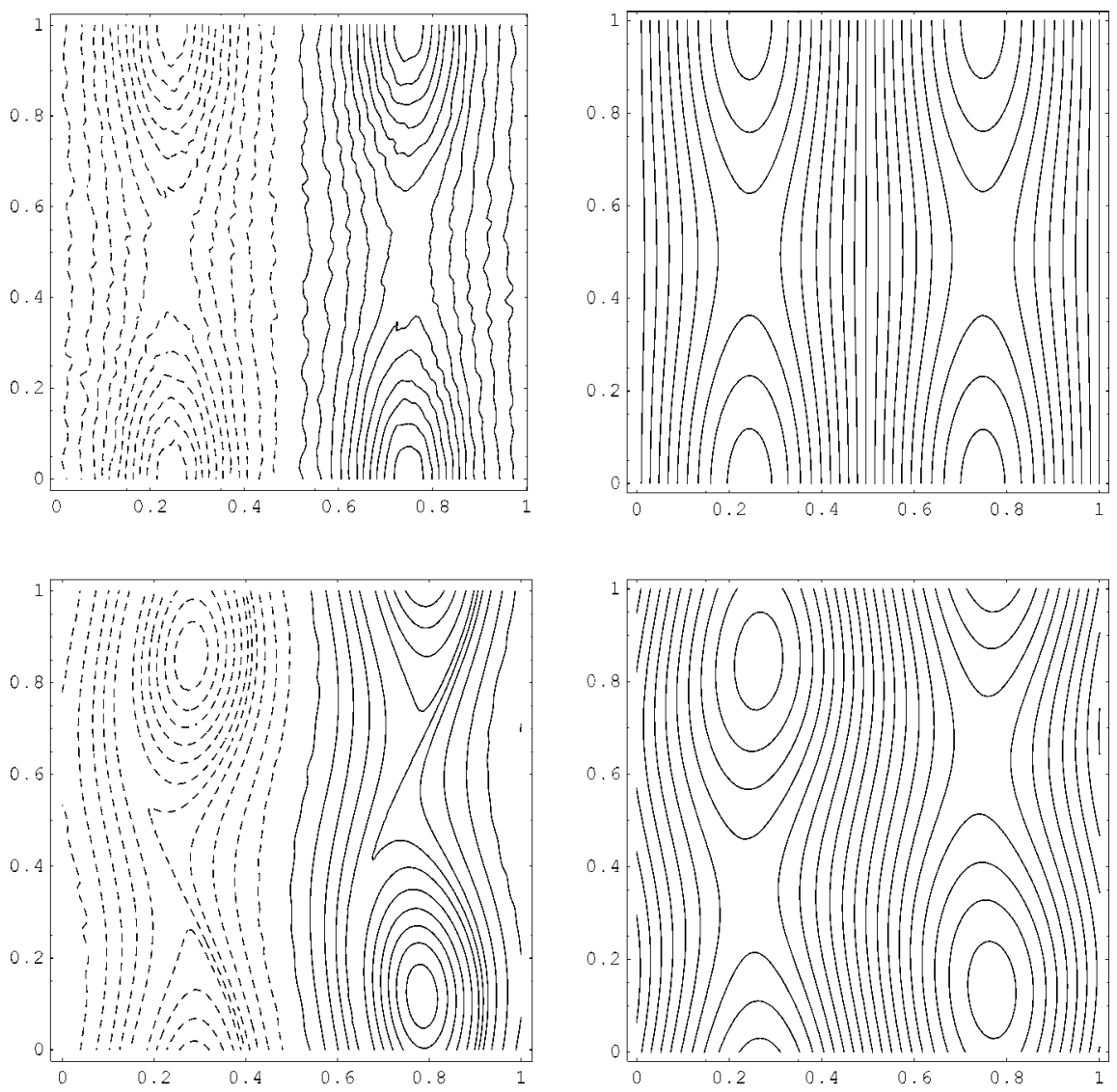

FIG. 9. Snapshots of vorticity (left) and stream function (right) in the evolution of the perturbed sn-dn dipole at time $=5,70$, and 245 of turnover units $\tau$ (from top). The system evolves to a round (diagonal-type) dipole.
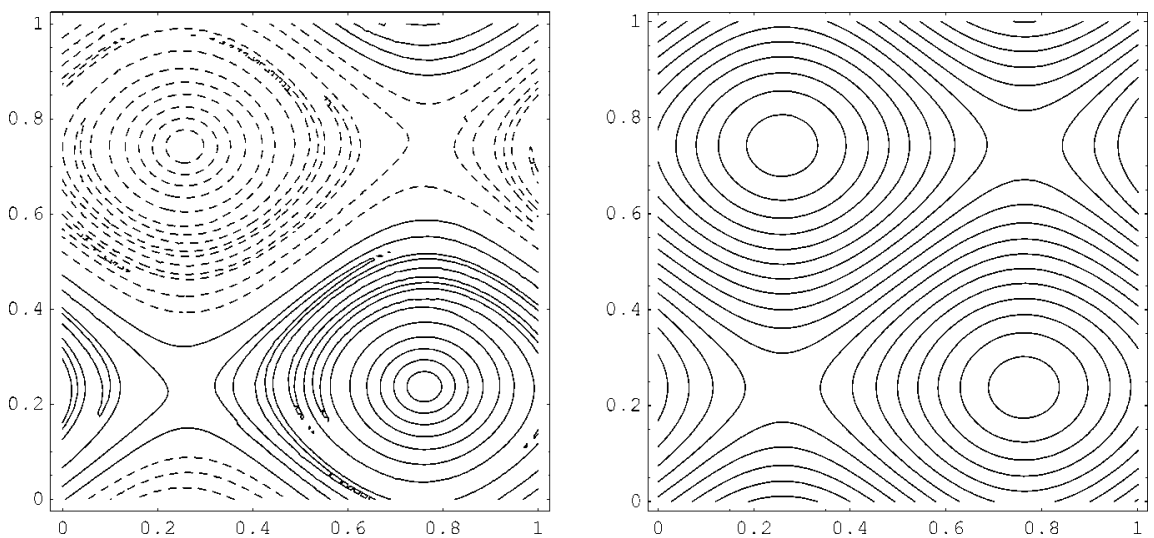

Euler equations. Such subgrid scales and processes are often represented (parametrized) as Navier-Stokes viscosity. Let us note, however, that the $2 \mathrm{D}$ viscosity has a different meaning from its three-dimensional counterpart. Most often it represents eddy viscosity rather than molecular viscous friction.

The numerical studies of decaying 2D turbulence reveal the formation of large-scale structures, which eventually relax to an equilibrium state (dipole, multipole, or bar). ${ }^{13}$ Some of these vortex structures closely resemble the doubly periodic solitons found theoretically.

It raises the problem of dynamic stability of these vortex solitons/arrays with respect to Eulerian or Navier-Stokes dynamics. Here we shall address it for two cases: soliton (5), called the sn-sn dipole here, and soliton (9), called the sn-dn dipole. The numerical simulations exploit a 2D semiLagrangian advective code developed by Gurarie.

\section{A. The sn-sn dipole}

We restrict our attention to a square geometry $\left(k=k_{1}\right)$. The range of moduli is then confined to the interval $0<k<3-\sqrt{8}$, and the period is $T=T_{x, y}=4 K(k) \sqrt{1-6 k+k^{2}}$. As $k$ increases the period decreases from the maximal value of $2 \pi$ to 0 , while the vortex cores narrow and strengthen. Hence, the parameter $k$ measures the degree of nonlinearity of such a soliton, $k=0$ corresponding to a linear (Fourier) eigenmode, while increasing $k$ yields strong, localized vortices (Fig. 5). 

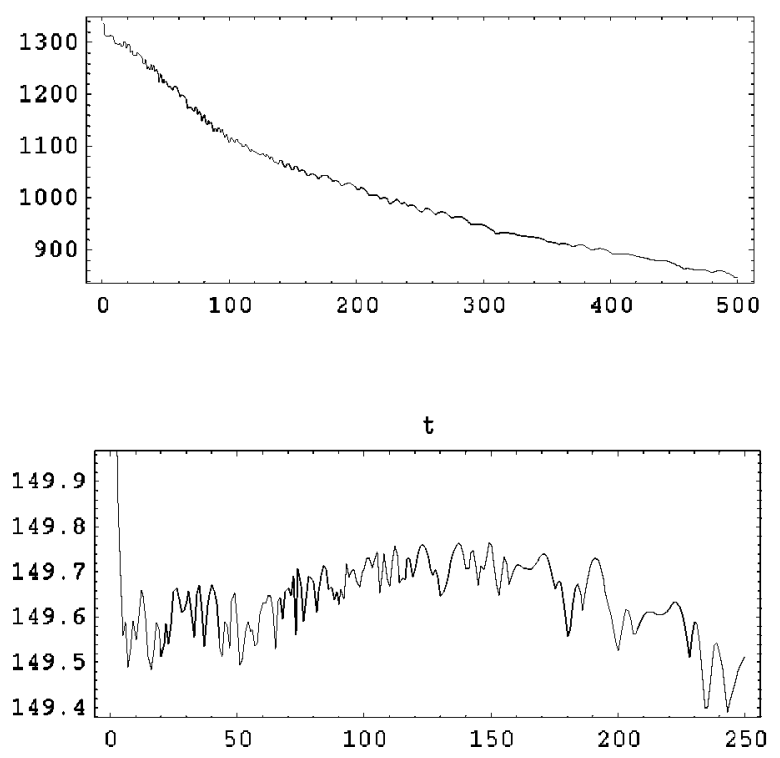
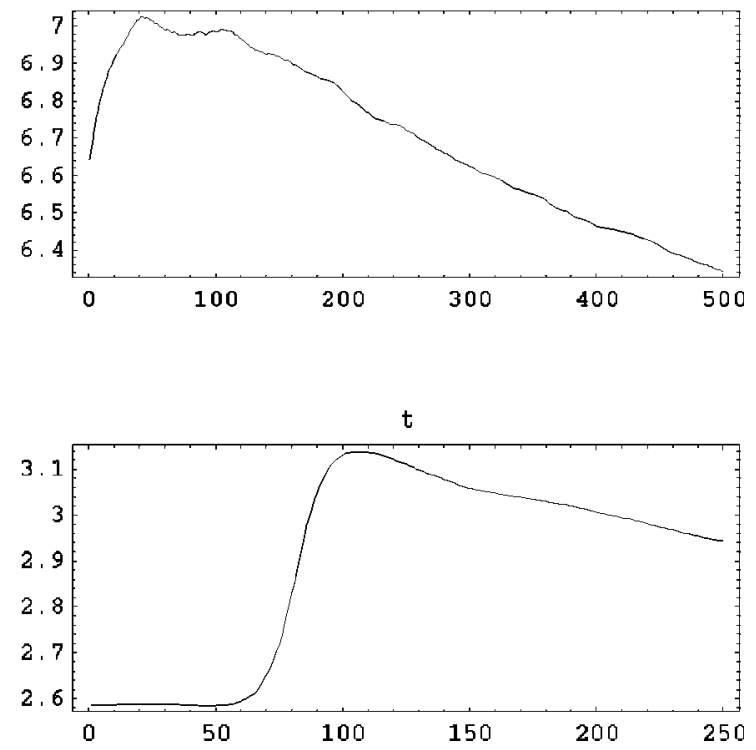

FIG. 10. The long-time evolution of maximal vorticity (left) and stream function (right) for both dipoles: (a) sn-sn and (b) sn-dn. The latter (b) undergoes a rapid transition from elongated/parallel to a round/diagonal configuration in the range of $70 \tau<t<100 \tau$.

\section{B. The sn-dn dipole}

Here, a square geometry confines the modulus $k$ to a range of $0.171<k<0.242$. The choice of $k=0.24\left(k_{1}\right.$ $=0.67)$ corresponds to two elongated counterrotating vortices inside parallel walls, as opposed to the diagonal orientation of a symmetric sn-sn dipole. This configuration is slightly more general than the Mallier-Maslowe vortices.

\section{Numerical code}

Here we provide only a brief description of the semiLagrangian advective code used for simulation. Further details will appear elsewhere. The code exploits material con- servation of vorticity along Lagrangian paths, and a suitable approximation of Lagrangian paths through velocity and acceleration of the flow. At each time step $\Delta t$ we compute the Lagrangian trajectories on a fixed grid, then advect the grid points backward in time, and estimate the vorticity at shifted grid points using spline interpolation of its values at nearby sites. While the code attempts to strictly conserve vorticity along Lagrangian paths (as one expects of an ideal Euler fluid), small-scale (subgrid) mixing creeps in due to interpolation. Let us note, however, that any physical 2D flow will always maintain some amount of local mixing, either through eddy viscosity, and/or other unresolved (subgrid)

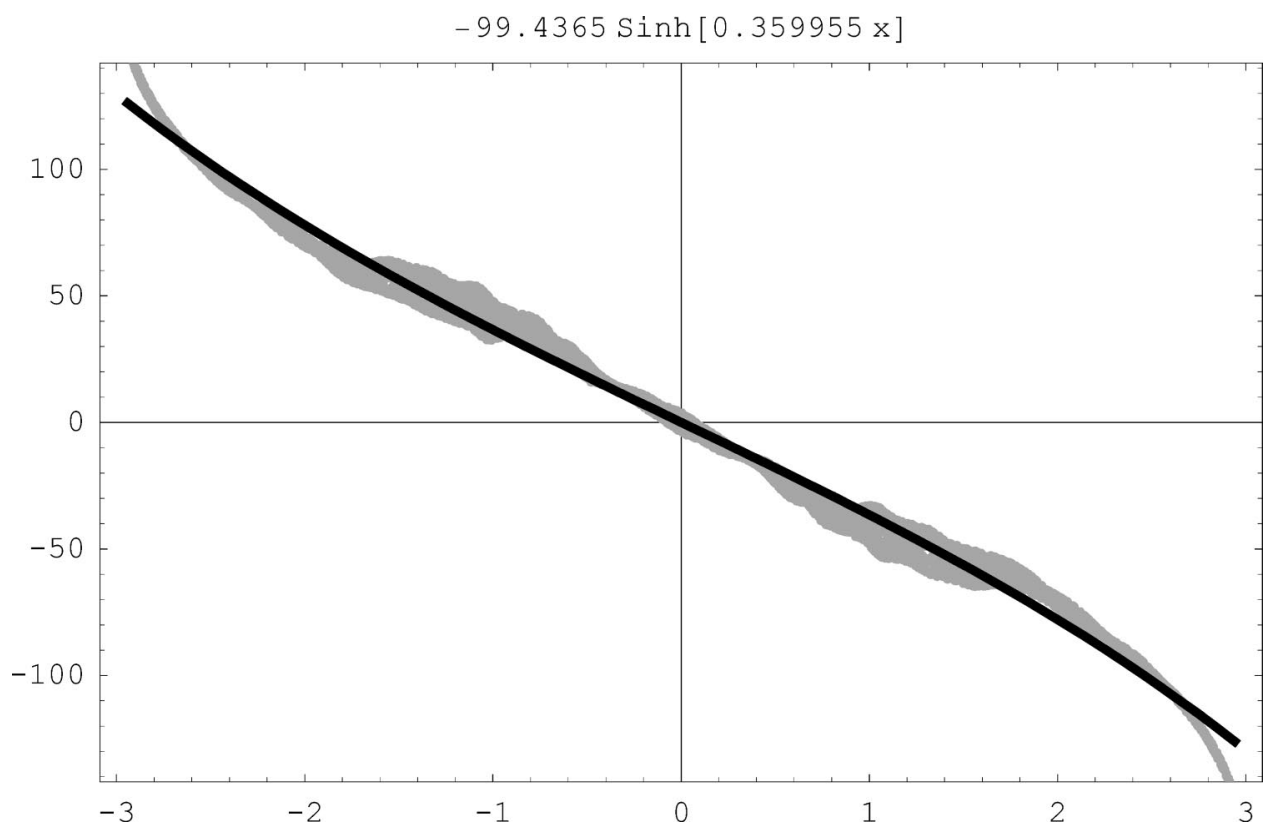

FIG. 11. Scatter plot and its sinh fit for the sn-dn soliton at a late (relaxed) state. One observes a "near-linear" (Fourier mode) dipole with a low $k$ $\approx 0.048$. 
processes. The code maintains one important feature of $2 \mathrm{D}$ turbulence, namely, the inverse cascade, ${ }^{14}$ that drives the system to a large-scale equilibrium. Similar to earlier NavierStokes works, ${ }^{13}$ most of our numeric experiments typically result in the dipole or bar states.

\section{Dipole perturbations}

We now discuss the detailed results for each case. The sn-sn dipole appears to be stable to all (even large) perturbations, as any (random) initial state tends to relax to a dipole equilibrium. Here we perturb the sn-sn dipole $(k=0.15)$ with a high-level random noise $50 \%$ of the maximum value of the initial vorticity), and run it on a $128 \times 128$ lattice of the unit square $0<x, y<1$. The flow equilibrates after several hundred turnover periods (defined here as $\tau=1 / \max |\zeta|$ at time $t=0)$. The relaxation process is demonstrated in Fig. 6 with three snapshots (top to bottom) at times $t=5 \tau, 40 \tau$, and $120 \tau$. The relaxed near equilibrium state $(t=500 \tau)$ has a vorticity-stream scatter plot that accurately fits the sinh profile, namely,

$$
\nabla^{2} \psi=-a \sinh (b \psi),
$$

with parameters $a=12.9, b=0.75$ (Fig. 7). Any solution of the latter is expressed through the basic sinh soliton (5) as

$$
\psi=\frac{1}{b} \psi_{S}[\sqrt{a b} x, \sqrt{a b} y \mid k(\sqrt{a b})] .
$$

We can estimate its period $T=\sqrt{a b}$ and modulus from the best-fit sinh curve and obtain $k=0.13$. It comes close to the initial value $k=0.15$, as if the nonlinear Euler dynamics has filtered out the initial random noise. The computed relaxed dipole closely resembles the sn-sn soliton at $k=0.13$. Figure 8 shows the relaxed quasiequilibrium on the left panel, and the exact $k=0.13$ soliton on the right panel. Furthermore, we estimated a relative-square-mean error between the computed $(t=500)$ and predicted (soliton) vorticity fields, and found a $6 \%$ error for $\zeta$, and a even smaller one (about $1 \%$ ), for their stream functions $\psi$.

Our second example, sn-dn dipole, tells a different story. It appears to be unstable to all (even small) initial perturbations. Here, we perturb it with a relatively low noise, $5 \%$ of the maximal value of the initial vorticity, and run over several hundred turnover periods (Fig. 9). The (elongated) parallel dipole structure is maintained for about 70 time units, after which it undergoes a rapid transformation to a diagonaltype (round) dipole state. Figure 9 demonstrates three snapshots of $\zeta$ and $\psi$ at points in times $t=5 \tau, 70 \tau$, and $245 \tau$. The transition occurs within the time range of $70 \tau<t<100 \tau$. One can see this transition in the long-time series of two diagnostic variables: the maximal vorticity (left panel) and the maximal stream function (right panel) of Fig. 10. The upper set (a) of Fig. 10 shows along-time series of sn-sn soliton and the lower one (b), the sn-dn soliton. Let us note that the maximal vorticity of the sn-dn plot (b) stays nearly constant, while its stream field undergoes a steep ascent in the range of 70-100 turnovers.

Once the terminal round dipole state is reached, the resulting quasiequilibrium strongly resembles a low modulus (near linear) sn-sn dipole. Indeed, the best sinh fit gives an estimate of the modulus as $k \approx 0.048$ (Fig. 11). Hence, longterm relaxation of the weakly perturbed sn-dn dipole drives it to a linear (Fourier) mode, $\psi_{0}(x, y)=\exp [i( \pm x \pm y)]$.

\section{CONCLUSIONS}

Two-dimensional turbulent flows have attracted recent attention, as robust monopole or dipole structures frequently emerge in the Navier-Stokes simulations. ${ }^{13,15}$ Indeed, similar vortices also arise in many experimental configurations in stratified or rotating fluids, ${ }^{16}$ or fluids under the influence of electromagnetic forces. ${ }^{17}$ The three-dimensional instabilities of the Mallier-Maslowe vortices have been studied as a model for the evolution of mixing layers and other free-shear flows. ${ }^{18}$ The instabilities depend on various factors, e.g., the symmetry properties of the perturbations and the Reynolds number of the ensuing Navier-Stokes simulations.

Here we review two classes of vortex solitons according to, or based on, their mathematical structure and the resulting flow configuration, and derive one new solution for each class. We study the dynamic stability of vortex arrays by choosing a typical member from each class for analysis. The issue of stability is addressed by simulating 2D Eulerian (or inviscid) flows with a semi-Lagrangian advective code. A sn-sn diagonal dipole, found earlier in the literature, is shown to be dynamically stable, and relaxes to a sinh-dipole state at a relatively high nonlinearity, i.e., strongly localized vortex pair. On the contrary, a sn-dn parallel dipole is unstable, and undergoes a transition to a round (diagonal-type) dipole with a fairly low value for the modulus $k$ of the associated Jacobi elliptic functions, i.e., nearly linear (Fourier mode) equilibrium. The evolution and stability of other periodic arrays in Sec. II will be addressed elsewhere.

Our work can be extended in other directions, and raises several issues. A first question concerns the role of a square geometry in maintaining soliton stability. Let us note that analytical solutions of Sec. II allow any rectangular geometry with an arbitrary aspect ratio, but the questions of their analytic structure and stability require further study. Some preliminary numeric results indicate that rectangular flows tend to relax to a different pattern-a parallel "bar state" (jets), ${ }^{13}$ rather than dipoles.

Comparing our numeric approach with earlier works, let us mark some important distinctions: unlike earlier works, ${ }^{13}$ we neither introduce Navier-Stokes viscosity per se, nor do we make a concerted effort to conserve vorticity contour dynamics. ${ }^{19}$ Rather, our code attempts to reproduce inviscid Eulerian dynamics with a subgrid mixing. The qualitative conclusions, however, are similar to the Navier-Stokes or contour advection approaches. Let us note that the relaxation time scales to equilibrium in our case are comparable with those obtained in earlier works. ${ }^{13,15}$ This suggests that largescale organization of 2D flows may be robust (insensitive) to its small-scale details and resolution. 


\section{ACKNOWLEDGMENTS}

Partial financial support has been provided by the Research Grants Council through Contract Nos. HKU 7067/ 00E and HKU 7006/02E.

${ }^{1}$ D. Montgomery, W. H. Matthaeus, W. T. Stribling, D. Martinez, and S. Oughton, "Relaxation in two dimensions and the sinh-Poisson equation," Phys. Fluids A 4, 3 (1992).

${ }^{2}$ R. Mallier and S. A. Maslowe, "A row of counter rotating vortices," Phys. Fluids A 5, 1074 (1993).

${ }^{3}$ R. A. Pasmanter, "On long-lived vortices in 2-D viscous flows, most probable states of inviscid 2-D flows and soliton equation," Phys. Fluids 6, 1236 (1994).

${ }^{4}$ T. Dauxois, "Nonlinear stability of counter-rotating vortices," Phys. Fluids 6, 1625 (1994).

${ }^{5}$ T. Dauxois, S. Fauve, and L. Tuckerman, "Stability of periodic arrays of vortices," Phys. Fluids 8, 487 (1996).

${ }^{6} \mathrm{~K}$. W. Chow, N. W. M. Ko, and S. K. Tang, "Solitons in $(2+0)$ dimensions and their applications in vortex dynamics," Fluid Dyn. Research 21, 101 (1997).

${ }^{7}$ K. W. Chow, N. W. M. Ko, R. C. K. Leung, and S. K. Tang, "Inviscid two dimensional vortex dynamics and a soliton expansion of the sinh-Poisson equation," Phys. Fluids 10, 1111 (1998).

${ }^{8}$ B. N. Kuvshinov and T. J. Schep, "Double-periodic arrays of vortices," Phys. Fluids 12, 3282 (2000).

${ }^{9} \mathrm{~K}$. W. Chow, "A class of doubly periodic waves for nonlinear evolution equations," Wave Motion 35, 71 (2002).

${ }^{10}$ K. W. Chow, S. C. Tsang, and C. C. Mak, "Another exact solution for two dimensional, inviscid sinh Poisson vortex arrays," Phys. Fluids 15, 2437 (2003).

${ }^{11}$ M. J. Ablowitz and H. Segur, Solitons and the Inverse Scattering Transform (SIAM, Philadelphia, 1981).

${ }^{12}$ M. Abramowitz and I. Stegun, Handbooks of Mathematical Functions (Dover, New York, 1964).

${ }^{13}$ Z. Yin, D. C. Montgomery, and H. J. H. Clercx, "Alternative statisticalmechanical descriptions of decaying two-dimensional turbulence in terms of 'patches' and 'points', " Phys. Fluids 15, 1937 (2003).

${ }^{14}$ D. Gurarie and S. Danilov, "Forced two dimensional turbulence in spectral and physical space," Phys. Rev. E 63, 061208 (2001).

${ }^{15}$ H. J. H. Clercx, S. R. Massen, and G. J. F. van Heijst, "Spontaneous spin-up during the decay of $2 \mathrm{D}$ turbulence in a square container with rigid boundaries," Phys. Rev. Lett. 80, 5129 (1998).

${ }^{16}$ E. J. Hopfinger and G. J. F. van Heijst, "Vortices in rotating fluids," Annu. Rev. Fluid Mech. 25, 241 (1993).

${ }^{17}$ O. Cardoso, D. Marteau, and P. Tabeling, "Quantitative experimental study of the free decay of quasi-two dimensional turbulence," Phys. Rev. E 49, 454 (1994).

${ }^{18}$ S. Julien, J. M. Chomaz, and J. C. Lasheras, "Three-dimensional stability of periodic arrays of counter rotating vortices," Phys. Fluids 14, 732 (2002).

${ }^{19}$ D. G. Dritschel and M. H. P. Ambaum, "A contour-advective semiLagrangian algorithm for the simulation of fine-scale conservative fields," Q. J. R. Meteorol. Soc. 123, 1097 (1997). 
Physics of Fluids is copyrighted by the American Institute of Physics (AIP). Redistribution of journal material is subject to the AIP online journal license and/or AIP copyright. For more information, see http:/ojps.aip.org/phf/phfcr.jsp

Copyright of Physics of Fluids is the property of American Institute of Physics and its content may not be copied or emailed to multiple sites or posted to a listserv without the copyright holder's express written permission. However, users may print, download, or email articles for individual use. 